\title{
Reflection-based Fiber Specklegram Sensor
}

\author{
L. Rodriguez-Cobo*, M. Lomer, R. Ruiz-Lombera, A. Cobo, J.M. Lopez-Higuera \\ Photonics Engineering Group, University of Cantabria, Av Los Castros S/N, Santander, Spain;
}

\begin{abstract}
In this paper, a Fiber Specklegram Sensor (FSS) based on Plastic Optical Fiber (POF) has been proposed with a reflectionbased configuration. A HeNe laser is launched through a coupler, reflected by a mirrored fiber end and detect the specklegram sequence using a commercial camera. Different sensor performance metrics have been analyzed in terms of final sensitivity to external vibration applied to the fiber. The results confirm the performance reduction of the reflectionbased scheme in comparison with the transmission-based approach but it can be also employed as sensing system where only a single fiber end is available.
\end{abstract}

Keywords: Speckle, POF, sensor, reflection, vibration, sensitivity

\section{INTRODUCTION}

Recently, fiber specklegram sensors have attracted considerable interest as a tool to measure physical and biochemical parameters. The fiber speckle pattern (specklegram) is generated by random interference between modes supported by the fiber when a coherent light propagates. The characteristics and properties of this phenomenon have been widely studied in the past [1-2], and have important applications in various disciplines. One of their key advantage is the high sensitivity of speckle patterns projected by an optical fiber. Considering that the total intensity of the speckle pattern is constant, each individual speckle varies slowly over time due to environmental variations surrounding the fiber, but the variation is more important when the fiber is directly perturbed by any mechanical effect (vibration, pressure, displacement...).

The spatial intensity variation in time of speckle contains information on the origin of the disturbance and can be analyzed to determine the correlation with the causing parameter. Therefore, fiber specklegrams can be used for sensing purposes. Since the 1980s, numerous designs and arrangements of sensors based on fiber specklegram have been proposed to measure vibration, displacement, strain, and acoustic waves [3-6]. These first sensors using bulky assemblies and signal processing was very extensive and laborious. However, during last years, technological advances allow to have affordable laser diodes and small CCD cameras [7-10]. Furthermore, computation technology have also been improved, making possible real time measurements. Most part of the proposed fiber specklegrams sensors work in transmission where the detection device is placed at the output end of the fiber. In some applications, these sensors are not a problem and have very good performance. However, for some scenarios, the idea of working only with a single fiber end (reflection) becomes more important. For these scenarios, the arrangement of the specklegram sensor would be similar to an optical reflectometer in the time domain (OTDR) configuration, but for this case, specklegrams projected by the multimode fiber are recorded by a CCD camera instead of a photodetector.

In this paper, a reflection-based fiber specklegram sensor is proposed and evaluated using plastic optical fiber (POF). Polishing the end of a POF and employing a drop of mercury pressured to the fiber end as reflector with about $70 \%$ reflection, and HeNe laser is launched through a coupler to be detected using a commercial CCD camera. Different properties of Fiber Specklegram Sensors (FSSs) are also analyzed to achieve a better understanding of the elements that have influence on the final sensor performance, particularly, its sensitivity to external perturbations.

\section{SPECKLE OPTICAL SENSITIVITY}

The sensitivity of a typical fiber specklegram sensor is basically given by the amount of modes and contrast of the dots (individual speckles) comprised within the captured images $[1,10]$. These two values are mainly influenced by three factors: the light source, the multimode fiber and the detecting device (usually a CCD camera). Employing a single detection device (e.g. CCD) and apart from the mechanical properties of the sensing fibers, the optical sensor sensitivity is strongly driven by the source properties and can be summarized into three main speckle pattern properties: contrast $\left(C_{S}\right.$, that is directly related to the coherence length of the laser source $\left.L_{C}\right)$; number of speckles $\left(N_{S}\right.$, that is inversely related to the squared value of wavelength) and pattern intensity $\left(I_{S}\right.$, that scales the speckles' differences and is related to the incoming

*luis.rodriguez@ unican.es; phone +34 942 200877; fax +34 942 200877; 
power and detector). For a given detection device and resolution and maintaining the multimode fiber, the final sensor sensitivity can be expressed as a function of:

$$
S_{S} \propto I_{S} \cdot C_{S} \cdot N_{S} \propto I_{S} \cdot \frac{L_{C}}{\lambda^{2}}
$$

This simplification assumes that the total intensity of the speckle pattern remains constant during the applied perturbations. The best approach is to record the whole speckle pattern to guarantee this condition; however, having enough individual speckles within the captured specklegrams may be sufficient for this requirement, simplifying the sampling requirements.

\section{RMS CONTRAST AND DIFFERENTIAL PROCESSING}

Each of the speckle pattern properties summarized in Eq. 1 should be experimentally analyzed in order to evaluate the performance of a reflection-based fiber specklegram sensor. Maintaining the laser source (same wavelength), the speckle optical sensitivity is mainly driven by its intensity $\left(I_{S}\right)$ that can be estimated as the mean intensity value of the captured specklegrams; and by each specklegram contrast $\left(C_{S}\right)$ that can be also quantified using the RMS contrast [10], defined as:

$$
C_{S} \approx C_{R M S}=\sqrt{\frac{1}{N \cdot M} \sum_{n=0}^{N-1} \sum_{m=0}^{M-1}\left(I_{n m}-\bar{I}\right)^{2}}
$$

where intensities $I_{n m}$ are the n-th m-th element of the two dimensional specklegram of size M by N. $\bar{I}$ is the average intensity of all pixel values of the specklegram.

Global speckle sensitivity has been empirically obtained employing the differential processing scheme [10], that can be defined for two consecutive specklegrams $\left(I_{n m}\right)$ of NxM pixels as follows (Eq. 3):

$$
D\{i\}=\frac{1}{K \cdot N \cdot M} \sum_{n=0}^{N-1} \sum_{m=0}^{M-1}\left|I_{n m}^{i-1}-I_{n m}^{i}\right|
$$

where $\mathrm{K}$ is the full scale value of the specklegram color map (e.g. $\mathrm{K}=255$ for 8-bit grayscale) and corresponds to the pixel of the $n, m$ position of the $i$-th specklegram. The differential intensity variation is proportional to the small variations caused by the perturbation between two consecutive specklegrams.

Differential sequence obtained with Eq. 3 exhibits the sensitivity of the global sensor that comprises both optical and mechanical sensitivities. Thus, the influence on the reflection-based setup (mechanical sensitivity) can be isolated from the optical sensitivity based on the experimental characterization.

\section{EXPERIMENTS AND RESULTS}

A reflection-based speckle setup has been employed to analyze the influence on its sensitivity only employing a single fiber end. Maintaining the laser source (HeNe laser), the detecting device (commercial USB webcam) and the plastic optical fiber ( $1 \mathrm{~mm}$ core diameter) light has been launched through the coupler to reach the free fiber while different reflections have been analyzed. In order to stablish a direct comparison with typical transmission schemes, the laser has been also launched by the fiber "free" end, maintaining the camera at the same location. This setup is depicted in Fig.1. 


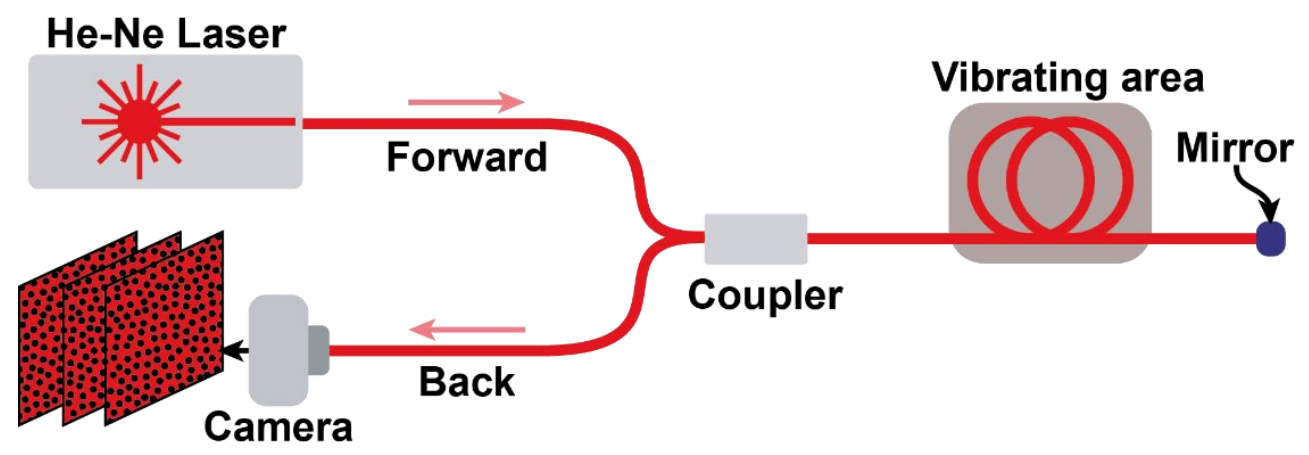

Figure 1. Experimental setup: A HeNe laser is launched through a 50:50 coupler to a multimode fiber whose free end is mirrored using a metallic surface. Light reflected at the end of the fiber travels back up to the detecting camera that records the specklegram sequence while a small fiber portion is under perturbation.

Some fiber length $(\sim 10 \mathrm{~cm})$ close to the free end has been attached to an eccentric motor that provides repeatable vibration, measured using an electric accelerometer to be of approximated frequency of $\sim 120 \mathrm{~Hz}$ and acceleration of $\sim 0.3 \mathrm{Gs}$. Firstly, the laser has been launched through the far end, working as a typical transmission-based specklegram sensor, to obtain the reference for comparison. After that, the laser has been moved to the coupler side, and a small quantity of mercury has been placed in direct contact to the polished surface of the POF far end. This mercury has been removed, leaving the polished end exposed to the air before inserting it into matched index liquid to isolate the reflection influence. An example of the different specklegrams obtained with each configuration is depicted in Fig. 2 (left).
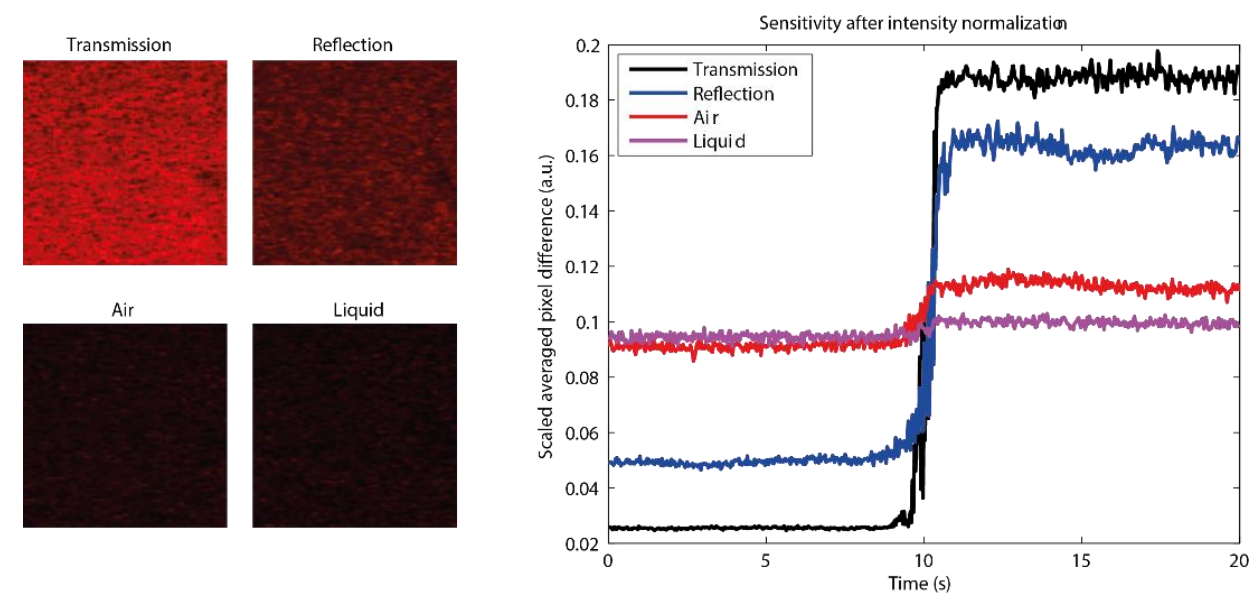

Figure 2. Example of 200x200 pixel specklegrams of each configuration (left). Differential sequence of each configuration during the vibration test (right). Each sequence has been scaled by its averaged intensity to allow a direct comparison.

Based on these configurations, a simple vibration test has been performed by applying 10 seconds of repeatable vibration to the same fiber portion, starting after 10 seconds of unaltered fiber to obtain the noise baseline. The differential processing method (Eq. 3) has been applied to obtain the sensitivity sequence (Fig. 2, right). Each differential sequence has been scaled using the averaged specklegram intensity of each configuration to allow a direct comparison.

The first configuration, working on transmission, has resulted as the best (as expected) based on its Signal-to-Noise Ratio (SNR), understood as the averaged differential value during the vibration minus the averaged value without vibration. However, the reflection-based configuration (mirroring the "free" end with mercury), exhibits also a remarkably good sensitivity reaching an experimental sensitivity of $\sim 70 \%$ compared to the transmission-based configuration, with a relative low baseline noise. On the other hand, when the fiber end is not mirrored, the resulting sensor is useless, exhibiting a very poor sensitivity and a high noise baseline.

Experimental sensitivity, the averaged RMS contrast, the averaged intensity and the baseline noise have been computed for each configuration, trying to understand the specific problems of a reflection-based implementation. These results have been normalized with the maximum of each parameter to make a direct comparison between configurations (Fig. 3). 


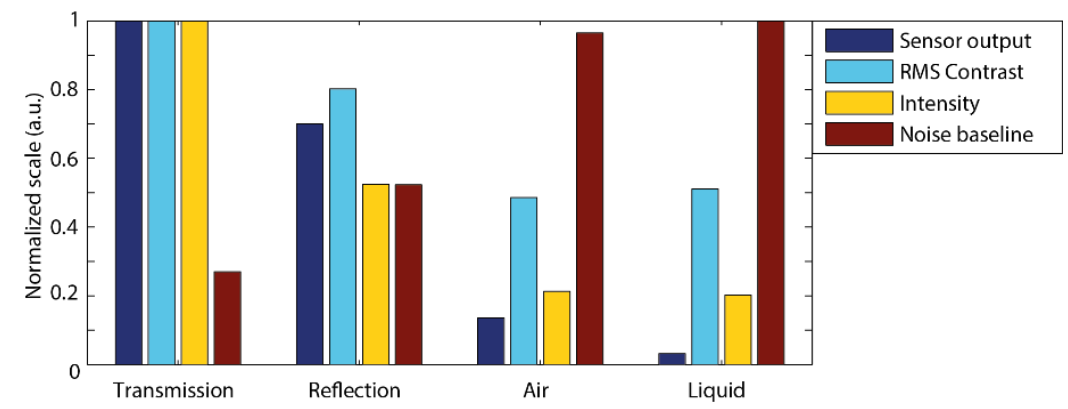

Figure 3. Experimental analysis of different parameters that have influence on the final performance. Each parameter has been scaled using the maximum of all the configurations.

The most remarkable parameter is the sensor output (Fig. 3, navy blue), defined as the ratio between the output amplitude using the differential processing scheme during vibration versus rest condition (noise baseline). The sensor output exhibits the maximum in the transmission-based setup and a reasonable drop when changing to the reflection-based configuration. Same conclusion can be obtained from the RMS contrast (Fig. 3, cyan) and averaged intensity (Fig. 3, yellow) of the achieved specklegrams, where the reflection configuration also maintains enough performance for sensing. On the contrary, the noise (Fig. 3, red) exhibits the maximum for the non-reflecting configurations (air and liquid) where the poor sensitivity, contrast and intensity achieved suggest that the perturbation may come through the coupler or other connection points that can be considered as noise. However, looking into the details of each parameter, the reflection based configuration exhibits a noise baseline higher than expected. This phenomenon can be explained due to the mirror effect created by the mercury in the polished fiber end that is not perfectly aligned to the fiber axis. These misalignments vary slightly the direction of the reflected light, making more difficult to keep propagating lower order modes, favoring higher order modes that increase the final sensitivity and noise.

\section{CONCLUSIONS}

In this paper, a proof-of-concept reflection-based fiber specklegram sensor has been demonstrated. Employing a CCD camera as detecting device, a HeNe laser has been launched through a coupler to a POF fiber with a polished end mirrored with mercury. Several parameters of fiber specklegram sensors have been also studied to determine the performance variation in terms of sensitivity and noise. Although the best achieved results were using the traditional transmission configuration, the reflection-based approach have been also proved as a reliable solution to simplify fiber specklegram sensors. This work has been supported by the project TEC2013-47264-C2-1-R.

\section{REFERENCES}

1. Goodman, J.W., Speckle Phenomena in Optics, Roberts and Company, Chapter 7, Englewood, Colorado, (2007).

2. Dainty, J.C., "Some statistical properties of random speckle patterns in coherent and partially coherent illumination", Opt. Acta, 17(10), 761-772 (1970).

3. Spillman, W.B.Jr., Kline, B.R., Maurice, L.B., and Fuhr, P.L., "Statistical-mode sensor for fiber optic vibration sensing uses", Appl. Opt., 28(3) 166-3176, (1989).

4. Yu, F.T.S., Wen, M., Yin, S., and Uang, C.M., "Submicrometer displacement sensing using inn-product multimode fiber speckle fields", Appl. Opt., 32(25) 4685-4689, (1993).

5. Pan, K., and Yu, T.T.S., "Temperature-compensated fiber specklegram strain sensing with an adaptive joint transformer correlator", Appl. Opt., 34(19) 3823-3825, (1995).

6. Chen, X., Yang, D., Lai, B., and Li, Z., "Acoustic Strain Effect of Multimode Optic-Fiber Sensor”, Proc. SPIE 2895, Fiber Optic Sensor V, 384-392 (1996).

7. Zhang, Z., and Ansari, F., "Fiber-optic laser speckle-intensity crack sensor for embedment in concrete", Sensors and Actuators A-126, 107-111 (2006).

8. Podbreznik, P., Donlagic, D., Lesnik, D., Cigale, B., and Zazula, D., "Cost-efficient speckle interferometry with plastic optical fiber for unobtrusive monitoring of human vital signs", J. Biomedical Optics, 18(10), 107001-8 (2013).

9. Rodriguez-Cobo, L., Lomer, M., Cobo, A., Lopez-Higuera, J.M, "Optical fiber strain sensor with extended dynamic range based on specklegrams," Sensors and Actuators A: Physical, 203, 341-345 (2013).

10. Rodriguez-Cobo, L., Lomer, M., Lopez-Higuera, J.M., "Fiber Specklegram Multiplexed Sensor," J. Lightwave Technology 32 (12), 2591-2597 (2015). 